\title{
Oval Cells: Potential Role in Liver Regeneration
}

\author{
Hussein Abdellatif ${ }^{1,2 *}$ \\ ${ }^{1}$ Department of Anatomy and Embryology, University of Mansoura, Egypt \\ ${ }^{2}$ Department of Anatomy, University of Bisha, Saudi Arabia
}

Received: January 06, 2018; Published: January 17, 2018

*Corresponding author: Hussein Abdellatif, Anatomy and Embryology Department, Faculty of Medicine, University of Mansoura, Mansoura, Egypt, Tel: +2 01145919955; Email: Hussein.abdullatif@hotmail.com

\begin{abstract}
The liver has the specific capacity to regulate its growth and repair. The healing process in the liver is characterized by the proliferation of all existing cell lines within the liver, including hepatocytes, epithelial cells that line the canaliculi, endothelial cells, and Kupffer and HSCs cells. The liver also contains "stem" cells or liver progenitor cells (oval cells in rodents) that can be activated by liver damage. Oval cells are the progeny of stem cells. They can divide rapidly but in contrast to stem cells do not possess the ability to self-renew. Oval cells have the potential to generate more than one differentiated cell type but cannot be serially transplanted. During liver regeneration, oval cells are essential at forming a second line of defense. In this article, we review some aspects of oval cells, characters, role in normal regenerative process as well as its role in liver diseases and malignancy besides its potential applications as source of cellular therapy.
\end{abstract}

Keywords: Oval cells; Liver regeneration; Hepatocytes; Stem cells

Abbreviations: AAF: Acetyl Amino Fluorine; AFP: Alpha-Feto Protein; ALD: Alcoholic Liver Disease; BMP4: Bone Morphogenetic Protein4; C/EBP $\alpha$ : C Enhancer Binding Protein Alpha; C/EBP $\alpha$ : CCAAT/Enhancer Binding protein $\alpha$; CK: Cyto keratin; CSC: Cancer Stem Cells; DDC: 3,5 Diethoxy carbonyl-1,4-Dihydro-Collidin; EpCAM: Epithelial Cell Adhesion Molecule; HNF4 $\alpha$ : Hepatocyte Nuclear Factor $4 \alpha$; IMS: Immuno Magnetic Sorting; OC: Oval Cells; OSM: Onco Statin M; PH: Partial Hepatectomy; SP: Side Population; T3: Triiodothyronine; TAT: Tyrosine Aminotransferase; TG: Transgenic; Thy-1: Thymus Cell Antigen 1; TNF: Tumor Necrosis Factor; TWEAK: TNF-like Weak Inducer of Apoptosis

\section{Introduction}

In the field of liver biology, the term liver stems cells or "hepatic stem cells have been defined and used by researchers in several different ways. The definitions can include, but may not be limited to the following:

a) Cells responsible for normal tissue turnover.

b) Cells which give rise to regeneration after partial hepatectomy $(\mathrm{PH})$.

c) Cells responsible for progenitor-dependent regeneration

d) Transplantable liver repopulating cells

e) Cells which result in hepatocyte and bile duct phenotype in vitro [1].

Hepatocyte turnover under the physiological condition is relatively slow which makes it quite difficult to investigate the cellular behavior in the course of homeo- static maintenance of the liver. Nevertheless, hepatocyte replacement does occur, and there must be some mechanism that ensures this tissue turnover.
One of the long-standing models is the so-called "streaming liver hypothesis" where a "flow" of hepatocytes is assumed just analogous to the well appreciated crypt-to-villi movement of the intestinal epithelial cells. In this model hepatocytes are newly formed in the periportal region and then gradually move, while undergoing lineage maturation, toward the central vein. Although appealing, much of the evidence accumulated so far argues against this hypothesis, and it is more favorably considered that the liver maintenance is rather achieved by simple division of the preexisting hepatocytes.

Very recently, however, a study in the human liver using mitochondrial mutations as a genetic marker identified that clonal patches of hepatocytes did emerge from the periportal regions and extended toward the central veins, supporting the presence of the hepatocyte flow as assumed by the streaming liver hypothesis [2]. The characteristic feature of the liver is its unique and remarkably high capacity to regenerate upon various injuries, such as those caused by partial hepatectomy or toxic insults. In rodent models, for example, after $70 \%$ partial hepatectomy, the liver can completely 
recover its initial volume and function within a week or so. During this recovery process, hepatocytes, as well as cholangiocytes, in the remaining liver undergo a few cycles of cell division to sufficiently restore the lost tissue. Thus, the liver regeneration can usually be achieved by proliferation of the differentiated, post mitotic hepatocytes that remain intact, without necessitating an involvement of stem/progenitor cell populations. When the liver suffers from severe and/or chronic damages, however, hepatocyte proliferation is suppressed.

It is under this condition when the facultative stem/progenitor cells are known to emerge and contribute to the liver regeneration process [3]. Those stem/progenitor cells referred to as oval cells in rodent models are characterized by their potentials to proliferate as well as to differentiate into hepatocytes and cholangiocytes, the two epithelial lineages in the liver. Although the term "oval cells" are used specifically in rodents, cells with similar characteristics have also been reported in various human liver diseases, such as chronic viral hepatitis, alcoholic liver disease (ALD), and nonalcoholic fatty liver disease (NAFLD), and are also implicated in tumorigenesis. In humans, these cells are usually referred to as "hepatic progenitor cells" or "Intermediate hepatobiliary cells" [4]. While oval cells are well known to emerge always from the periportal area, the cellular origin of oval cells is still not clarified. Ever since their initial characterization, phenotypic resemblance between oval cells and bile duct epithelial cells has suggested that they presumably originate from the biliary tree. The fact that most of the molecular markers for oval cells are also expressed in cholangiocytes supports this notion.

It is not clear, however, whether most if not all cholangiocytes can equally or similarly behave as progenitors for oval cells or there is a certain type of specialized "origin-of-oval cells" located somewhere among cholangiocytes. Potentially lying on an extension of the latter possibility is the model that the canal of Hering, a structure where interlobular bile ducts and hepatocytes are connected, is the origin of oval cells [5]. Given its anatomical location in between cholangiocytes and hepatocytes, it appears reasonable to assume that this structure may serve as a niche for putative stem cells for these two cell lineages. Unfortunately, a direct proof for this model is hampered by lack of any specific marker for cells constituting the canal of Hering. Identification of such a molecule and a subsequent genetic lineage tracing study should help clarifying this issue. Although a possible contribution of hepatocytes as an origin of oval cells can also be considered, a study using mice with chimeric livers have suggested that this is not likely the case [6].

As a matter of course, emergence and expansion of oval cells upon liver injury is not an autonomous process within these cells but involves various other types of cells which interact either directly or indirectly with oval cells and also possibly with their putative precursor cells and together shape the entire phenomenon often termed as "oval cell response". Mesenchymal cells such as stellate cells have long been suggested to physically interact with oval cells and exert some signals on them. A recent study has highlighted that a population of mesenchymal cells expressing thymus cell antigen (Thy-1) which is distinct from stellate cells or myofibroblasts reside in close proximity to oval cells in rat liver. Further characterization of this unique population may provide a clue to understand the nature of signals controling oval cell behaviors [7].

\section{Oval Cell Activation by Different Injury Models}

Chronic injury conditions in the liver are usually associated with induction of inflammation and the role of lymphocytes and inflammatory responses have been suggested. In accord with this notion, several inflammatory cytokines, such as tumor necrosis factor (TNF)-alpha and interferon-gamma, have been shown to modulate oval cell response, although their modes of action remain not fully clarified. A cytokine well appreciated to be involved in oval cell regulation is TNF-like weak inducer of apoptosis (TWEAK); transgenic mice over expressing TWEAK in the liver exhibit periportal oval cell hyperplasia, while DDC diet-induced oval cell expansion was significantly reduced in mice lacking the Tweak receptor Fn14 as well as in wild-type mice administrated with a blocking anti-TWEAK monoclonal antibody. As a signal related to oval cell response, recent studies have identified activation of the canonical Wnt/ beta-catenin pathway in oval cells. The Wnt/betacatenin pathway is well known to be involved in stem cell regulation in various organs and tissues, and further characterization of the role of this pathway in oval cells, including its relevant target genes and interaction with other signaling pathways is awaited [8].

\section{Oval Cells Characterization}

Oval cells were initially described by Farber, using a rat model of liver carcinogenesis as "small oval cells about the ducts and vessels in the portal areas" having "scanty, lightly basophilic cytoplasm and pale blue staining nuclei (by hematoxylin and eosin stain [9]. Since then, many studies have further characterized these cells and have established them as facultative liver stem/progenitor cells that are likely to play a relevant role in liver regeneration from various types of injuries Thus, oval cells are considered to be capable of differentiating into two hepatic epithelial lineages, i.e., hepatocyte and cholangiocyte. In possible relation to this notion, oval cells express both hepatocyte (Albumin) and cholangocyte (CK19) markers. The immature hepatocyte marker alphafetoprotein (Afp) is known to be expressed in oval cells in rats, but not in mice. Similarly, expression of the hepatoblast marker Dlk1 has been shown in a subpopulation of rat oval cells but is not found in mouse oval cells [10]. There are several monoclonal antibodies that have long been used as "golden standards" to recognize oval cell markers, such as OV-1 and OV-6 in rats and A6 in mice. OV-1 antibody reacts with an unknown antigen expressed on the surface of oval cells and thus can be used to isolate these cells, while OV-6 antibody recognizes a common epitope in the cytoskeleton components CK14 and CK19.

Unfortunately, the A6 antibody used for mouse studies recognizes some intracellular antigen and thus is not suitable to be used for sorting of viable oval cells. Similar to the situation with fetal liver hepatoblasts, much effort has been made in recent years to explore cell surface molecules that can be used to identify and isolate oval cells. This has led to the identification of EpCAM and CD133 (also known as prominin1) as novel oval cell markers 
in both mice and rats [11]. The oncofetal protein glypican-3 has also been documented as a rat oval cell marker. Notably, however, these molecules as well as the OC-1/OC-6 and A6 antigens are all expressed also in cholangiocytes in the normal liver. This fact strongly implies a close relationship between cholangiocytes and oval cells as mentioned earlier with the former possibly being an origin of the latter. Interestingly, Trop2 (Tacstd2) a transmembrane molecule that is structurally related to EpCAM has been found to be expressed exclusively in oval cells in the injured liver, but not in cholangiocytes in the normal liver [11].

Thus Trop2 may serve as a genuine "oval cell marker" and would be advantageous for further characterization of oval cells. Similarly, a recent study identified a transcription factor, Foxl1, as another oval cell-specific marker [12]. Although this molecule is not a cell-surface antigen, a transgenic (Tg) mouse line expressing the Cre recombinase under the control of the Foxl1 promoter has been made and proven to be quite useful. Thus, a lineage tracing study using these Foxl1-Cre Tg mice demonstrated that both hepatocytes and cholangiocytes were found as descendants of Foxl1+ oval cells. This does not necessarily indicate that single oval cells can clonally differentiate into these two lineages but strongly supports the notion that oval cells are bipotential progenitors for hepatocytes and cholangiocytes. A recent study (Table 1) has established a panel of surface reactive monoclonal antibodies that can each detect different populations of ductal and periductal cells in the oval cell response. Intriguingly, some of them seem to label cell populations that are apparently enriched or reside specifically in the oval cell induced livers with little or no reactivity shown in the normal liver [13]. Identification of the corresponding antigen molecules as well as further characterization of these cell populations should expedite our understanding of the mechanisms of the oval cell response at the cellular and molecular levels.

Table 1: Commonly used markers for the identification of LPCs (oval cells) in rodents.

\begin{tabular}{|c|c|c|}
\hline Cell & Marker & Location \\
\hline Adult hepatocyte related & Albumin, alpha anti trypsin, hepatic nuclear factor 4 (HNF4), Cytokeratin 8, 18 & Intracellular \\
\hline Fetal hepatocyte related & AFP, MPK, glutathione s Transferase, Fn14, or TWEAK receptor, CD13, CD24, CD29. & CD26, CD49F, ATP binding cassette G2 \\
\hline Adult biliary related & Cytokeratin 7, 19, OV-6, A6 antigen, OV1, EpCAM, CD133, C-met. & Thy 1, c-Kit, stem cell antigen, CXCR4, CD34 \\
\hline Adult haematopiotic related & Chromogranin A, parathyroid hormone related peptide, neurotropin receptors. & Intracellular cell membrane \\
\hline Neuroepithelial related & TROP2, connexin 32 & Cell membrane \\
\hline Progenitor related & & Cell membrane \\
\hline
\end{tabular}

\section{Oval Cell Isolation and In Vitro Assay}

Using flow cytometry-based cell separation methods in combination with the aforementioned cell surface markers, oval cells can be viably isolated and subjected to in vitro culture to evaluate their proliferation and differentiation potentials. In general, oval cells isolated from injured livers proliferate to form colonies in vitro in the presence of certain combinations of growth factors and the clonally expanded cells are capable of differentiating into both hepatocyte and cholangiocyte lineages under appropriate culture conditions. These results strongly suggest that oval cells indeed possess clonal bi-lineage differentiation potential, at least in vitro, a notion which needs to be evaluated using in vivo experimental systems as well. As oval cell antigens like EpCAM and CD133 are also expressed in cholangiocytes under uninjured conditions, the cells positive for these markers were also isolated from normal adult livers and similarly subjected to in vitro culture experiments [11].

Several methods have been employed for the isolation of oval cells (OC). These techniques range in complexity and include Nicodenze gradient separation, and sorting by flow cytometric (FACS) or immunomagnetic sorting (IMS). FACS had been the gold standard for isolating OC prior to the introduction of IMS. IMS has become a widely used method for separating different cell types. It has numerous advantages compared to other methods. It is a convenient, easy to use system which can yield enrichment of up to 99\% depending on the specificity of the cell surface marker used. Stem cell biology has benefited significantly from the introduction of magnetic cell sorting [14]. Thy-1+Cell Sorting: Thy-1 (CD90) is a GPI-anchored membrane glycoprotein of the Ig super family which is involved in signal transduction and it is expressed by a wide spectrum of hematopoietic stem and progenitor cells as well as non hematopoietic cells including neurons, endothelium at inflammatory sites and hepatic OC. High purity OC enrichment is achieved through IMS using the OC surface marker Thy-1. Using Thy-1 in conjunction with FACS sorting, a 95-97\% enriched population of OC may be obtained.

Prior to sorting, the NPC population must be isolated by the simple gravity enrichment method. Thy-1+ cells from rat liver can be isolated using indirect labeling strategies. Currently fluoresce in isothiocyanate (FITC)-conjugated mouse anti-rat CD90 (Thy-1.1) has been employed to positively select OC [15]. Immuno-magnetic Sorting: IMS technology is based on the use of microbeads, columns, and a magnetic field. The microbeads are super-paramagnetic particles (approximately $50 \mathrm{~nm}$ in size, biodegradable, and nontoxic to cells), which are coupled directly or indirectly to specific monoclonal antibodies. These beads magnetically label the target cell population. The patented column technology is specifically designed to generate the high-strength magnetic field that is required to retain the labeled cells, while maintaining optimal cell viability and function. By placing the column in a permanent magnet (called the "separator"), the magnetic force is sufficient to retain the target cells. By simply rinsing the column with buffer, all the unlabeled cells are washed away. Once the column is detached from the magnet, the labeled fraction can be eluted [16]. 


\section{In Vitro Assay}

Several laboratory techniques may be used to characterize OC. Cytospins from OC suspensions can be employed for immunohistochemistry or immunofluorescence. RNA, DNA or protein can be extracted and processed for molecular biology. Additionally, OC may be cultured for in vitro assays. Several media have been proposed to culture OC with varying degrees of success. It is very difficult to maintain and expand OC in culture without spontaneous differentiation. In vitro assays are very useful in analyzing the properties of OC and their response to exogenous factors. In particular, two properties are critical to the OC phenotype: proliferation following certain types of liver injury and migration from the periportal space to the liver parenchyma. Trafficking, mobilization and homing of OC are multifactorial processes that are regulated by several factors including adhesion molecules, cytokines and chemotactic molecules. Therefore, proliferation and migration assays may be useful in clarifying the molecular mechanisms underlying OC activation [17].

\section{Proliferation Assay}

It may be used to assess the effects of a specific factor on oval cell proliferation. Briefly, the factor is added at different dosages to the basic OC medium (IMDM). Growth kinetics of the OC are evaluated at different time points (usually at 1, 3, 5, and 7 days). A positive control (FBS 10\%) and a negative control (bovine serum albumin, BSA, 0.5\%) are required. Every determination should be performed at least in triplicate [18].

\section{Migration Assay}

It may be used to assess the effects of a specific factor on oval cell motility. Briefly, oval cells (OC) are seeded on the microporous membrane of a transwell. The factor is added at different dosages to the basic oval cell medium in the lower chamber. This will result in the migration of oval cell through the membrane, following the chemo attractive factor gradient. Oval cells motility can be assessed at different time points [18].

\section{Differentiation Capacity of Oval Cells}

The characteristic feature of the liver stem/progenitor cells is their potential to differentiate into two lineages, i.e., hepatocytes and cholangiocytes. In addition to these two hepatic cell lineages, much evidence has been accumulated supporting that the liver stem/progenitor cells are also capable of differentiating into pancreatic and other cell lineages both in vitro and in vivo under appropriate experimental settings. A number of experimental models have been established in order to induce oval cells proliferation. Oval cells can therefore be isolated and utilized in investigations into the mechanisms governing differentiation towards hepatocytes or cholangiocytes. Studies have also focused on elucidating the key factors important for oval cells differentiation towards hepatocytes and extracellular growth factors have been shown to be a key factor. He et al. [16] demonstrated that, in vitro oval cells differentiated into mature hepatocytes in the presence of epidermal growth factor (EGF) and hepatocyte growth factor (HGF) using a two-step induction protocol which first changed the oval cells into small hepatocytes then differentiated them further to exhibit morphological, phenotypic and functional characteristics of hepatocytes. Okaya et al. [19] showed that treatment of the rat oval cell line 0C15-5 with Oncostatin M (OSM) induced differentiation into hepatocytes as observed by alterations in morphology in which microvilli appeared and a large cytoplasm developed with organelles as well as the expression of hepatocyte markers such as albumin and tyrosine aminotransferase (TAT) [20].

Following 2-acetylaminofluorene/partial hepatectomy (AAF/ $\mathrm{PH}$ )-induced liver injury, the rat livers displayed a linear relationship between OSM-receptor (OSM-R) expression and the number of oval cells and OSM-R expression was exclusively found in oval cells. More recently, bone morphogenetic protein 4 (BMP4), a member of the transforming growth factor super-family has been shown to induce rat hepatic progenitor cell differentiation towards the hepatocyte lineage in vitro as identified by reverse transcriptase polymerase chain reaction (RT-PCR) and western blotting analysis of mature liver markers. In addition, the administration of the primary hepatocyte mitogen triiodothyronine (T3) was shown to accelerate the differentiation of oval cells into hepatocytes in vivo. Induction of hepatic differentiation by BMP4 and T3 coincided with stimulated expression of specific intracellular liver enriched transcription factors CCAAT/enhancer binding protein $\alpha(\mathrm{C} / \mathrm{EBP} \alpha)$ and hepatocyte nuclear factor $4 \alpha(\mathrm{HNF} 4 \alpha)$, respectively, both of which have been suggested in previous studies to be important in the onset of the hepatocyte differentiation program in oval cells [21].

\section{Role of C/EBP- $\alpha$ in Oval Cells Differentiation to Hepato- cytes}

C/EBP $\alpha$ is a member of the CCAAT/enhancer binding protein family and is capable of interacting with CCAAT motifs present in numerous gene promoters C/EBP $\alpha$ expression is rapidly induced during liver injury once the differentiation process is nearly complete. This relates to the paradigm that C/EBP $\alpha$ may have a role in terminal differentiation of hepatocytes through regulation of promoter and enhancer sequences of several important liver genes involved in hepatic glycogen synthesis, gluconeogenesis (e.g. tyrosine amino transferase) and lipid homeostasis [22]. Furthermore, in contrast to increased C/EBP- $\alpha$ expression being fundamental in hepatocyte differentiation, Yamasaki et al., 2006 have postulated that the absence of C/ EBP $\alpha$ in cholangiocytes has an indirect effect by inducing the expression of the genes HNF6 and HNF1 $\beta$, both of which have important regulatory roles in cholangiocyte differentiation. Therefore, C/EBP- $\alpha$ expression could be important in the decision between hepatocytes and cholangiocytes from bipotential precursors [23].

HNF4 $\alpha$ is important in Hepatocyte Morphogenesis: There is conflicting evidence for the role of the hepatocyte nuclear factor HNF4 $\alpha$ during differentiation of oval cells to hepatocytes. Dabeva et al., 1995 investigated differentiation of oval cells in vivo by studying the expression of liver enriched transcription factors (Including: HNF $1 \alpha$; Foxa $\alpha, \beta, \gamma ; \operatorname{HNF} 4 \alpha ; \mathrm{C} / \mathrm{EBP} \alpha, \beta, \delta$ ) following non-carcinogenic [D-galactosamine (GaIN) treatment] induced liver injury in rats [24]. Dabeva and colleagues, 1995 suggested that because of controlling HNF4 $\alpha$ and HNF1 $\alpha$ by a higher order locus, it is possible that in the oval cell differentiation program, 
when HNF1 $\alpha$ is already expressed, activation of high HNF4 $\alpha$ levels are not indicated [24]. However, the normal program of hepatocyte differentiation is suspected to be similar to that of oval cells, as developing liver cells (hepatoblasts) may correspond to immature progenitor cells, which are maintained after birth and constitute a minor subpopulation in the adult liver giving rise to the oval cells during liver injury.

In normal development, HNF4 $\alpha$ may act upstream in a cascade to activate other hepatocyte transcription factors and thus there is no compensation by other factors, as many nuclear hormone receptors, such as HNF4 $\alpha$ are involved in chromatin remodelling via interaction with transcriptional co-activators. In addition, from a morphological perspective, development of the normal liver architecture is crucial for correct liver function. HNF $4 \alpha$ has been proved to be essential in regulating epithelial morphogenesis and maturational differentiation of hepatocytes. Suetsugu et al., 2008 demonstrated in vitro that Overexpression of HNF4 $\alpha$ induced a mature gene expression pattern and liver functions in fetal hepatic progenitor cells, which when transplanted into mice with liver fibrosis (i.e. in vivo) improved survival.

This study highlights that although in vitro work is beneficial to identifying key factors important for HPC (oval cells) hepatocyte differentiation, further complementary in vivo work is ultimately required as other extracellular environmental factors may influence oval cells differentiation [25]. The review by Erker and Grompe 2008 emphasises the importance of factors secreted by surrounding cells types, such as inflammatory, Kuppfer and hepatic stellate cells in stimulating oval cells proliferation and differentiation [26]. Indeed, differentiation of oval cells into mature hepatocytes has been shown to be induced by hepatic stellate cells [27].

Differentiation into Non Hepatic Lineages: The liver and the pancreas share a common developmental origin and a bipotential precursor cell population for these organs has been identified within the embryonic endoderm. In addition, hepatocytes and pancreatic beta-cells are known to have similarities in gene expression profiles and possess similar inherent glucose sensing systems thereby being capable of responding to changes in blood glucose concentrations. Consistent with these facts, many studies have demonstrated that liver stem/progenitor cells from both embryonic and adult origins as well as hepatocytes can be converted to insulin producing cells, functional pancreatic beta-cell-like cells, and/or to islet-like cell clusters containing other pancreatic lineages under certain conditions. Clonally expanded H-CFU-C derived from fetal mouse liver show expression of pancreatic endocrine and exocrine lineage markers in culture and can be integrated into and formed pancreatic ducts and acinar cells when transplanted into pancreas of recipient mice. Notably, H-CFU-C is also shown to be capable of differentiating into gastric and intestinal cells in vivo. Purified adult rat hepatic oval cells can be differentiated into pancreatic endocrine hormoneproducing cells when cultured in a high-glucose environment [28].

The Role of Oval Cells (Hepatic Progenitor Cells) in Liver Fibrosis and Cirrhosis: Recent experiments have clearly demonstrated that there is an association between fibrosis and progenitor cell activation. Although the mechanisms that link liver progenitor cells and fibrogenic cells are as yet unknown, there are now several signalling pathways in common operating during their differentiation in liver repair and regeneration. Whether these signaling pathways are activated in the different cell types independently in parallel or whether there is cross-talk remains unclear [27].

The Ductular Reaction (Oval Cell Response) and Fibrosis: The ductular reaction is the term given to a duct-like proliferation of a population of liver progenitor cells in the setting of fibrosis. The extent of the ductular reaction appears to correlate directly with impaired hepatocyte replication consistent with the notion that progenitor cells are only recruited when replication of mature liver cells is inhibited. Multiple investigators have shown a compelling direct association between the extent of the ductular reaction and the severity of fibrosis in cases with chronic liver diseases suggesting that progenitor cell activation plays an important if undefined role in fibrosis.

A detailed marker analysis of ductular reactions in livers from cirrhotic cases demonstrated that these ductular reactions had a bipolar structure with bidirectional differentiation to either hepatocytic or biliary lineages [29]. Other groups have not observed mutually exclusive expression of hepatocytic and biliary markers during progenitor cell proliferation but instead describe "intermediate hepatocytes" that express both sets of markers. These intermediate hepatocytes appear in contiguity with ductular progenitor cells when liver inflammation and necrosis is moderate or severe suggesting that differentiation of progenitor cells (oval cells) towards the hepatocytic lineage is directly related to the extent of parenchyma injury [30]. Regardless of the details of progenitor cell activation and marker expression, these observations strongly support the concept that hepatic progenitor cells (oval cells) of the ductular reaction contribute directly to the fibrogenic process, most likely through cell-cell cross talk between the progenitor cells and nearby mesenchymal populations and via recruitment of inflammatory cells [31]. The signals that mediate progenitor cell quiescence and activation, however are still unclear. Nguyen et al., 2007 made the seminal observation that rat liver progenitor cells (oval cells), unlike hepatocytes are resistant to the growth inhibitory effects of the growth factor TGF-B providing at least a partial mechanistic basis for their differential activation in the TGF-B rich environment of fibrosis and cirrhosis [32].

The Source of Progenitor Cells in Fibrosis: Understanding of the complex origins of progenitor cells and fibrogenic cells is still rudimentary. On the one hand, there is data in the literature demonstrating that progenitor cells from both the liver and the bone marrow can become hepatic stellate cells and myofibroblasts in fibrosis. On the other hand, some groups suggest that stellate cells are themselves progenitor cells and play an important role in parenchyma and non parenchyma cell reconstitution in the injured liver. Both concepts remain controversial and questions about the quantitative importance of each and the relevant stimuli and signaling pathways have not yet been investigated [33].

Oval Cells (Progenitor Cells) as a Source of Myofibroblasts: Myofibroblasts defined operationally as fibrogenic cells expressing $\alpha$-smooth muscle actin ( $\alpha$-SMA) are the primary source of fibrillar 
collagens and other abnormal extracellular matrix (ECM) proteins in fibrosis. Hepatic stellate cells, a heterogeneous population of vitamin A-storing cells normally located in the space of Disse have long been considered the major source of myofibroblasts in fibrosis although other resident myofibroblast precursor cells including portal fibroblasts and mesenchymal cells are increasingly recognized as providing equal if not more significant contributions [33]. The embryonic origin of hepatic stellate cells and portal fibroblasts is debated although studies using transgenic mice suggest that hepatic stellate cells as well as certain perivascular mesenchymal cells originate from the mesoderm of the septum transversum [34].

Portal fibroblasts and other mesenchymal cells appear during early development during which time stellate cells and portal fibroblasts share at least one specific marker (p75NTR). Whether progenitor cells also contribute to the population of stellate cells, portal fibroblasts and other myofibroblasts in the setting of significant or chronic injury in the adult liver is less clear. Bone marrow transplants have been touted as a potential therapy for chronic liver disease thus understanding the relationship between bone marrow stem cells and matrix-producing cells in fibrosis is particularly important. There are now multiple reports suggesting that progenitor cells from the bone marrow can migrate to the injured liver and differentiate into stellate cells and myofibroblasts. A variety of studies, most involving whole bone marrow transplantation but at least one in which the clonal progeny of a single hematopoietic stem cell were transplanted yielded similar evidence that marrow derived progenitor cells could generate fibrogenic myofibroblasts [35]. A more recent study by Higashiyama et al. [36] however showed no evidence that any collagen expression in the injured liver was from bone marrowderived cells. Kallis and Forbes, 2009 summarized the existing studies suggesting that heterogeneity in the population of bone marrow derived stem cells explains these divergent results [37].

Oval cells and Liver Cancer: There is now compelling evidence that oval cells/HPCs are at the very least bipotent, capable of giving rise to both hepatocytes and cholangiocytes. The fact that oval cell activation (ductular cell reaction) precedes the development of HCC in almost all models of hepatocarcinogenesis and invariably accompanies chronic liver damage in humans makes it almost certain that the mature hepatocyte is not the cell of origin of all HCCs; indeed, perhaps only a small minority of HCCs are derived from the mature hepatocyte [38]. The fact that oval cells/HPCs can be infected with $\mathrm{HBV}$ is also consistent with a possible histogenesis of HCC from such cells. An origin of HCC from HPCs is often inferred from the fact that many tumours contain an admixture of mature cells and cells phenotypically similar to HPCs. This would include small oval-shaped cells expressing OV-6, CK7 and 19 and chromogranin-A, along with cells with a phenotype intermediate between HPCs and the more mature malignant hepatocytes [39].

Cells with an HPC phenotype have also been noted in a relatively rare subset of hepatic malignancies where there are clearly two major components, an HCC component and a cholangiocarcinoma component again suggestive of an origin from a bipotential progenitor. Cells resembling HPCs (eg OV-1+ or OV-6+) have also been noted in hepatoblastoma, the most common liver tumour in childhood, likely to be stem cell-derived given there can be both epithelial and mesenchymal tissue components. Hepatoblastomas can even have structures mimicking embryonic intrahepatic bile duct formation with the formation ductal plate-like structures [40]. Direct evidence of a role for oval cells in the histogenesis of HCC can be obtained experimentally. Dumble et al. isolated oval cells from p53-null mice and when the cells were transplanted into athymic nude mice they produced HCCs [38]. A probable origin of HCC from oval cells is suggested by the fact that if oval cell expansion is blocked in the CDE diet mouse model by targeting c-Kit with imatinib mesylate, then tumour formation is reduced. If tumours do arise from oval/HPCs, then this would suggest a block in oval cell differentiation, a process termed 'stem cell maturation arrest'. This hypothesis is supported by the fact that HCCs induced by a CDE diet exhibit a range of neoplastic phenotypes recapitulating stages in normal development, suggesting transitional states between bipotent oval cells and hepatocytes [41]. Along these lines of thought, four prognostic subtypes of HCC have been identified, corresponding to a hierarchy of liver cell lineages. Those with the poorest prognosis possessed a sizeable proportion of EpCAM + AFP + cells (hepatoblast- like) or EpCAM- AFP + cells (oval cells like), whereas those with EpCAM-AFP-cells (mature hepatocyte- like) or EpCAM + AFP- cells (cholangiocyte-like) had a more favourable outcome. Moreover, gene expression profiling has identified a subset of HCCs with a poor prognosis that have a profile consistent with an origin from HPCs/ oval cells and simple enumeration of CK19-positive cells in HCC can identify a group who have a shorter time to recurrence [42].

Oval Cells, Cancer Stem Cells and Side Population Cells: There is a growing realization that many, if not all, cancers contain a minority population of self-renewing stem cells, the cancer stem cells (CSCs) which are entirely responsible for sustaining the tumor as well as giving rise to proliferating but progressively differentiating cells that are responsible for much of the cellular heterogeneity that is so familiar to histopathologists. Many liver tumors probably have their origins in normal liver stem cells, particularly HPCs but do liver tumors have CSCs? With the development of non-obese diabetic/severe combined immunodeficient (NOD/SCID) mice for the xenografting of human tumours came the first good in vivo evidence for the existence of CSCs in leukaemia, breast and brain tumours. This assay has been criticized as simply reflecting the ability of human cells to grow in a foreign, inappropriate murine microenvironment and as such the cells should be called 'tumourinitiating cells' (TICs), rather than CSCs.

Whatever the limitations of the assay, several markers have been advocated with which to prospectively isolate human HCC CSCs [43]. Chiba and colleagues, 2006 have reported that two of the four HCC cell lines that they studied had SP cells comprising $0.25 \%$ and $0.8 \%$ of the cell population. These cells were highly proliferative and relatively resistant to apoptosis in vitro [44]. Microarray analysis indicated that several genes implicated in 'stemness', e.g. WNT pathway genes were substantially up-regulated in the SP cells in comparison to non- SP cells. Using the 'gold-standard' NOD/SCID 
mouse assay for CSCs, they found that transplanting $10^{3}$ liver SP cells consistently yielded tumors, whereas transplantation of $10^{6}$ non-SP cells failed to give rise to tumors so are SP cells in HCC the CSCs? They could be in some cases? Prominin-1 (CD133) a pent span membrane protein whose function is as yet unclear, has been suggested as a CSC marker in many different tumours, including colon, pancreas, brain and prostate although its utility has recently been called into question. Using the CD133/1 antibody, a number of studies have suggested that the CD133-positive fraction enriches for HCC CSCs.

As might be expected of CSCs, they appear as a minority $(<2 \%)$ population in primary tumours, although continued passaging has resulted in considerable enrichment of CD133+ cells in some HCC cell lines, up to $90 \%$ [45]. HCCs with higher than the median number (1.32\%) of CD133-positive cells are correlated with shorter survival, higher recurrence rates and higher tumour grade and CD133+ cells appear highly resistant to conventional therapeutic drugs, such as 5-FU and doxorubicin [45]. Tang et al., 2008 have suggested that HCC CSCs are descendents of normal parenchymal stem/progenitor cells that have lost sensitivity to the inhibitory growth effects of TGF $\beta$ while cell selection based on Thy-1 (CD90), a disputed marker of oval cells, in combination with CD44 has also produced cells with aggressive tumorigenic potential. It remains to be seen how much overlap there is between these various markers, or whether there is a 'one-fits-all' marker for CSCs in HCC and indeed in other tumour types as well [46].

\section{Future perspectives}

At present, orthotopic liver transplantation is the most commonly used procedure to treat various liver diseases.This, however has always been hampered by persistent shortage of donor organs. Although isolated mature hepatocytes when transplanted have been shown to successfully repopulate the recipient liver with considerably high efficiency at least in rodent models, the rates of engraftment and survival of transplanted hepatocytes in human liver is often very limited. Due to their large diameter (20-40lm), up to $70 \%$ of transplanted hepatocytes get trapped in the hepatic sinusoids which leads to temporary obstruction with subsequent portal hypertension, poor engraftment rate and finally the demand for a high amount of transplantable cells (up to $2 \times 10^{7}$ hepatocytes in rodent models).

For this reason, alternative administration of progenitor cells is considered to be a promising future treatment option for numerous acute or chronic liver diseases [47]. At present, research has succeeded in obtaining transplantable progenitor/stem cells from liver, bone marrow, umbilical cord blood, Whartons's jelly stem cells, skin and adipose tissue. Few approaches have been developed to reduce the rejection of transplanted cells and to improve the poor cell engraftment rate in order to reduce the overall required number of cells to administer. One proposed method to decrease rejection rate and to increase engraftment rate of transplanted cells is the (co) administration of mesenchymal stem cells; not only due to their proven immunomodulatory and immunosuppressive properties, but also because they may provide an appropriate pericellular and extracellular environment [48].

\section{Acknowledgement}

We thank Anatomy and Embryology Department, Faculty of Medicine, University of Mansoura, Egypt. For the support and follow up.

\section{References}

1. Grompe M (2003) Pancreatic-hepatic switches in vivo. Mechanisms of Development 120(1): 99-106.

2. Fellous TG, Islam S, Tadrous PJ, Elia G, Kocher HM, et al. (2009) Locating the stem cell niche and tracing hepatocyte lineages in human liver. Hepatology 49(5): 1655-1663.

3. Michalopoulos GK, De Frances MC (1997) Liver regeneration. Science 276(5309): 60-66.

4. Fausto $N$ (2004) Liver regeneration and repair: hepatocyte progenitor cells and stem cells. Hepatology 39(6): 477-1487.

5. Paku S, Schnur J, Nagy P, Thorgeirsson SS (2001) Origin and structural evolution of the early proliferating oval cells in rat liver. The Am J of Path 158(4): 1313-1323.

6. Wang $\mathrm{X}$, Willenbring H, Akkari Y, Yumi Torimaru, Mark Foster, et al. (2003) Cell fusion is the principal source of bone-marrow-derived hepatocytes. Nature 422(6934): 897-901.

7. Yovchev MI, Zhang J, Neufeld DS, Grozdanov PN, Dabeva MD (2009) Thymus cell antigen-1- expressing cells in the oval cell compartment. Hepatology 50(2): 601-611.

8. Strick-Marchand H, Masse GX, Weiss MC, Di-Santo JP (2008) Lymphocytes support oval cell- dependent liver regeneration. J Immunol 181(4): 2764-2771.

9. Farber E (1956) Similarities in the sequence of early histological changes induced in the liver of the rat by ethionine 2-acetylaminofluorene and 3-methyl 4 dimethylaminoazobenzene. Cancer Res 16:142-148.

10. Jelnes P, Santoni-Rugiu E, Rasmussen M, Friis S, Nielsen JH, et al. (2007) Remarkable heterogeneity displayed by oval cells in rat and mouse models of stem cell mediate liver regeneration. Hepatology 45(6): 14621470.

11. Okabe M, Tsukahara Y, Tanaka M, Suzuki K, Saito S, et al. (2009) Potential hepatic stem cells reside in EpCAM+cells of normal and injured mouse liver. Development 136(11): 1951-1960.

12. Sackett SD, Li Z, Hurtt R, Gao Y, Wells RG, et al. (2009) Foxl1 is a marker of bipotential hepatic progenitor cells in mice. Hepatology 49(3): 920-929.

13. Dorrell C, Erker L, Lanxon-Cookson KM, Abraham SL, Victoroff T, et al. (2008) Surface markers for the murine oval cell response. Hepatology 48(4): 1282-1291.

14. Schmitz B, Radbruch A, Kümmel T, Wickenhauser C, Korb H, et al. (1994) Magnetic activated cell sorting (MACS)-a new immunomagnetic method for megakaryocytic cell isolation: comparison of different separation techniques. Eur J Haematol 52(5): 267-275.

15. Petersen BE, Zajac VF, Michalopoulos GK (1998) Hepatic oval cell activation in response to injury following chemically induced periportal or pericentral damage in rats. Hepatology 27(4): 1030- 1038.

16. Mason DW, Williams AM (1980) the kinetics of antibody binding to membrane antigens in solution and at the cell surface. Biochem J 187(1): $1-20$.

17. Piscaglia AC, Shupe TD, Oh SH, Gasbasrini A, Petersen BE (2007) Granulocyte-Colony Stimulating Factor Promotes Liver Repair and Induces Oval Cell Migration and Proliferation in Rats. Gastroenterology 133(2): 619-631.

18. Hatch HM, Zheng D, Jorgensen ML, Petersen BE (2002) SDF 1alpha/ CXCR4: a mechanism for hepatic oval cell activation and bone marrow stem cell recruitment to the injured liver of rats. Cloning Stem Cells 4(4): 339-351. 
19. He ZP, Tan WQ Tang YF, Feng MF (2003) Differentiation of putative hepatic stem cells derived from adult rats into mature hepatocyte in the presence of epidermal growth factor and hepatocyte growth factor. Differentiation 71(4-5): 281-290.

20. Okaya A, Kitanaka J, Kitanaka N, Satake M, Kim Y, et al. (2005) Oncostatin $M$ inhibits proliferation of rat oval cells OC15-5inducing differentiation into hepatocyte. Am J Pathol 166(3): 709-719.

21. Fan JH, Shen H, Dai QM, Minuk GY, Burzynski FJ, et al. (2009) Bone morphogenetic protein- 4 induced rat hepatic progenitor cell (WB-F344 cell) differentiation toward hepatocyte lineage. J Cell Physiol 220(1): 7281.

22. Nerlov C (2010) Transcriptional and translational control of C/EBPs: the case for "deep" genetics to understand physiological function. Bioessays 32(8): 680-686.

23. Yamasaki H, Sada A, Iwata T, Niwa T, Tomizawa M, et al. (2006) Suppression of C/EBP alpha expression in periportal hepatoblasts may stimulate biliary cell differentiation through increased Hnf6 and Hnf1b expression. Development 133(21): 4233-4243.

24. Dabeva MD, Hurston E, Shafritz DA (1995) Transcription factor and liver specific messenger RNA expression in facultative epithelial progenitor cells of liver and pancreas. Am J Pathol 147(6): 1633-1648.

25. Suetsugu A, Nagaki M, Aoki H, Motohashi T, Kunisada T, et al. (2008) Differentiation of mouse hepatic progenitor cells induced by hepatocyte nuclear factor-4 and cell transplantation in mice with liver fibrosis Transplantation 86(9): 1178-1186.

26. Erker L, Grompe M (2008) Signaling networks in hepatic oval cell activation. Stem cell research 1(2): 90-102.

27. Chen Y, Jurgens K, Hollemann T, Claussen M, Ramadori G, et al. (2003) Cell-autonomous and signal-dependent expression of liver and intestine marker genes in pluripotent precursor cells from Xenopus embryos. Mech Dev 120(3): 277-288.

28. Yang L, Li S, Hatch H, Ahrens K, Cornelius JG, et al. (2002) In vitro transdifferentiation of adult hepatic stem cells into pancreatic endocrine hormone-producing cells. Proc Natl Acad Sci 99(12): 8078-8083.

29. Zhou H, Rogler LE, Teperman L, Morgan G, Rogler CE (2007) Identification of hepatocytic and bile ductular cell lineages and candidate stem cells in bipolar ductular reactions in cirrhotic human liver. Hepatology 45(3): 716-724.

30. Katoonizadeh A, Nevens F, Verslype C, Pirenne J, Roskams T (2006) Liver regeneration in acute severe liver impairment: a clinicopathological correlation study. Liver Int 26(10): 1225-1233.

31. Ruddell RG, Knight B, Tirnitz-Parker JE, Akhurst B, Summerville L, et al. (2009) Lymphotoxin-beta receptor signaling regulates hepatic stellate cell function and wound healing in a murine model of chronic liver injury. Hepatology 49(1): 227-239.

32. Nguyen LN, Furuya MH, Wolfraim LA, Nguyen AP, Holdren MS, et al. (2007) Transforming growth factor-? Differentially regulates oval cell and hepatocyte proliferation. Hepatology 45(1): 31-41.

33. Li Z, Dranoff JA, Chan EP, Uemura M, Sevigny J, et al. (2007) Transforming growth factor-beta and substrate stiffness regulate portal fibroblast activation in culture. Hepatology 46(4): 1246-1256.
34. Villeneuve J, Pelluard-Nehme F, Combe C, Carles D, Chaponnier C, et al. (2009) Immunohistochemical study of the phenotypic change of the mesenchymal cells during portal tract maturation in normal and fibrous (ductal plate malformation) fetal liver. Comp Hepatol 8: 5.

35. Miyata E, Masuya M, Yoshida S, Nakamura S, Kato K, et al. (2008) Hematopoietic origin of hepatic stellate cells in the adult liver. Blood 111(4): 2427-2435.

36. Higashiyama R, Moro T, Nakao S, Mikami K, Fukumitsu H, et al. (2009) Negligible contribution of bone marrow-derived cells to collagen production during hepatic fibrogenesis in mice. Gastroenterology 137(4): 1459-1466.

37. Kallis YN, Forbes SJ (2009) the bone marrow and liver fibrosis: friend or foe? Gastroenterology 137(4): 1218-1221.

38. Dumble ML, Croager EJ, Yeoh GC, Quail EA (2002) Generation and characterization of p53 null transformed hepatic progenitor cells: oval cells give rise to hepatocellular carcinoma. Carcinogenesis 23(3): 435445 .

39. Libbrecht L, Roskams T (2002) Hepatic progenitor cells in human liver diseases. Semin Cell Dev Biol 13(6): 389-396.

40. Gornicka B, Ziarkiewicz-Wroblewska B, Michalowicz B, Pawlak J, Wroblewski T, et al. (2001) Immature hepatic tumor of bimodal differentiation in a young adult patient: a novel lesion expressing betacatenin and mimicking a distinct phase of hepatogenesis. J Hepatol 34(6): 955-961.

41. Hixson DC, Brown J, McBride AC, Affigne S (2000) Differentiation status of rat ductal cells and ethionine-induced hepatic carcinomas defined with surface-reactive monoclonal antibodies. Exp Mol Pathol 68(3): 152-169.

42. Durnez A, Verslype C, Nevens F, Fevery J, Aerts R, et al. (2006) The clinicopathological and prognostic relevance of cytokeratin 7 and 19 expression in hepatocellular carcinoma A possible progenitor cell origin. Histopathology 49(2): 138-151.

43. Burkert J, Wright NA, Alison MR (2006) Stem cells and cancer: an intimate relationship. J Pathol 209(3): 287-297.

44. Chiba T, Kita K, Zheng YW, Yokosuka O, Saisho H, et al. (2006) Side population purified from hepatocellular carcinoma cells harbors cancer stem cell-like properties. Hepatology 44(1): 240-251.

45. Ma S, Chan KW, Hu L, Lee TK, Wo JY, et al. (2007) Identification and characterization of tumorigenic liver cancer stem/progenitor cells. Gastroenterology 132(7): 2542-2556.

46. Tang Y, Kitisin K, Jogunoori W, Li C, Deng CX, et al. (2008) Progenitor/ stem cells give rise to liver cancer due to aberrant TGF-beta and IL-6 signaling. Proc Natl Acad Sci U S A 105(7): 2445-2450.

47. Sandhu JS, Petkov PM, Dabeva MD, Shafritz DA (2001) Stem cell properties and repopulation of the rat liver by fetal liver epithelial progenitor cells. Am J Pathol 159(4): 1323-1334.

48. Santoni-Rugiu E, Jelnes P, Thorgeirsson SS, Bisgaard HC (2005) Progenitor cells in liver regeneration: molecular responses controlling their activation and expansion. APMIS 113(11-12): 876-902.

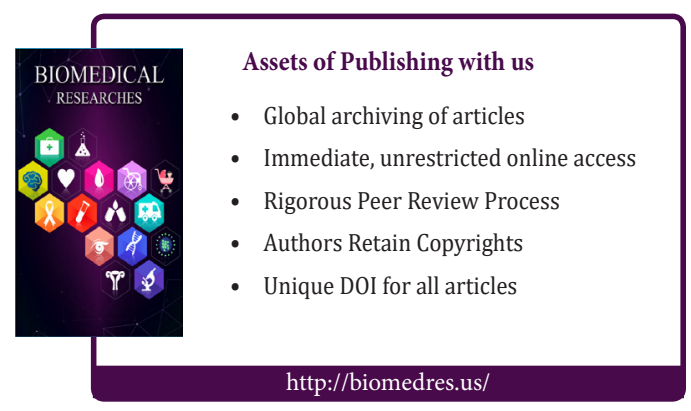

